\title{
The role of olfactory ensheating cells in regenerative medicine: review of the literature
}

\author{
Andreea Grosu-Bularda' ${ }^{1}$, Claudiu Manea ${ }^{2}$, loan Lascar ${ }^{1}$ \\ ${ }^{1}$ Department of Plastic and Reconstructive Surgery, Emergency Clinical Hospital, Bucharest, Romania \\ ${ }^{2}$ ENT\&HNS Department, „Sfanta Maria“ Hospital, Bucharest, Romania
}

\section{ABSTRACT}

Olfactory ensheathing cells (OECs) join olfactory axons in their entrance to the central nervous system, representing a unique population of glial cells with functions in olfactory neurogenesis, axonal growth and olfactory bulb formation. Olfactory ensheathing cells have a great potential to induce repair for neural injuries, in central nervous system and peripheral nervous system, existing numerous experimental and clinical studies lately, reporting beneficial effects in anatomical and functional recovery. Studies are also conducted in order to establish possible pro-regenerative effects of the OECs, their potential in tissue repair and ability to modulate the immune system. The aim of this paper was to review the properties of olfactory ensheathing cells and their potential therapeutic role in regenerative medicine.

This paper is supported by the Sectoral Operational Programme Human Resources Development (SOP HRD,) financed from the European Social Fund and by the Romanian Government under the contract number POSDRU/159/1.5/S/137390

KEYWORDS: Olfactory ensheathing cells, cell transplantation, nerve recovery, neural injuries, immunomodulation

\section{INTRODUCTION}

The olfactory system represents a special area in the human body where neurons are replaced, neurogenesis being possible during the lifetime. Olfactory ensheathing cells (OECs), also known as Olfactory ensheathing glial cells or Olfactory ensheathing glia, represents the glial cells of the olfactory nervous system that ensheath the non-myelinated axons of olfactory neurons in a similar manner of Schwann cells that ensheath non-myelinated peripheral neurons. OECs represent an unique glial cell type which distributes in both central nervous system and peripheral nervous system (migrating from the peripheral nervous system into the central nervous system), characteristic that distinguishes those cells from typical glia ${ }^{1}$.

The property of OECs to create a favourable microenvironment for neurogenesis justifies their use for cellular transplantation in injuries of the central nervous system and peripheral nervous system in order to obtain nerve recovery and increasing the axonal regrowth $^{2,3}$.

In addition to their role in stimulation of neural repair, there are also other properties of the olfactory ensheathing cells which are investigated for the potential usage in regenerative medicine: modulation of critical aspects of tissue repair through structural remodelling and support, implication in glial scars reorganization, remyelination, angiogenesis properties, phagocytic role, metabolizing toxic macromolecules, enhancement of neurotrophic and antigenic stimuli, and modulation of the immune response $\mathrm{e}^{4}$.

\section{THE ROLE OF THE OECS IN OLFACTORY SYSTEM}

Primary olfactory receptor neurons originate from stem cells residing in the epithelium of the nasal cavity. Their axons extend through the basal side of the epithelium and gather into fascicles (axon bundles) and form the olfactory nerve, highly branched, which ends in the olfactory bulb within the brain ${ }^{5}$. During the lifetime, in the olfactory epithelium, neurogenesis represents a continuous process: the new formed axons grows and repair the first cranial nerve after injuries, not the axons that regenerate from mature cells ${ }^{6}$. The life span of olfactory neurons is around 1-3 months. Then, neurogenesis occurs, apoptotic neuronal cells being replaced by new sensory neurons originating from the basal layer of the olfactory epithelium ${ }^{7}$.

An important role in neurogenesis is attributed to olfactory ensheathing cells, glial cells that arise from neural crest (derived from the olfactory placode dur- 
ing development $)^{8}$. Olfactory ensheathing cells of the olfactory epithelium accompany the axons of primary olfactory neurons in their path to the olfactory bulbs. OECs have the ability to guide axonal outgrowth: trough their migration, OECs influence the axon motility; increasing the motility of olfactory ensheathing cells results in improving extension of the axons and stimulates the activity of the growth cone ${ }^{9}$. The ability of OECs to migrate from the peripheral nervous system into the central nervous system is critical for the development of the olfactory system and also for the neural repair after injuries. When the olfactory neuroepithelium is damaged, stem cells from the mucosa differentiate and repair it, accompanying the glial cells up to the level of the olfactory bulb. Being ensheathed by the OECs, the olfactory axons can grow and access the central nervous system; this property is not seen in axons that regenerate after injuries of the dorsal roots; they fail to enter too far into the nervous tissue of the spinal cord ${ }^{10}$. It was shown that adhesion molecules L1/neuron-glia cell adhesion molecule (L1/Ng-CAM) and neural cell adhesion molecule (N-CAM), both present in the plasma membranes of ensheathing cells and immature olfactory receptor neurons, help the olfactory axons to use the glial cell surfaces as a growth substratum ${ }^{6}$. OECs have the ability to synthesize different proteins: laminin, protein involved in cell adhesion, nexin and nerve growth factor, both being involved in promoting neural growth ${ }^{6}$. In the structure of the olfactory nerve OECs surround large bundles of axons. The olfactory nerve passes from the peripheral nervous system through the cribriform plate to enter the central nervous system. The primary olfactory axons defasciculate, separates into specific subtypes and then project to their correct topographical targets. The OECs are intermixed with the axons, having different arrangement and morphology compared to OECs in the peripheral nerve. The nerve fiber have an outer and an inner layer, in both layers we can find OECs. OECs have different role in nerve fiber layers: in the outer layer, OECs permit defasciculation in the mixed bundles of axons, while in the inner nerve fiber layer OECs are involved in segregation of axons and refasciculation, depending on their type of odorant receptor. In the peripheral nervous system, OECs induce adhesion of cells and fascicular organisation of axons, while in the olfactory bulb those cells promote different cellular functions ${ }^{5,11,12}$. Regeneration of the olfactory system occurs continuously during adulthood, which is a different situation from what it happens in the central nervous system. OECs play key roles in this regeneration, through releasing of diffusible factors to attract neural progenitors and regulate their proliferation and differentiation ${ }^{13}$.

Glial cells of the peripheral nervous system have phagocytic properties, being involved in protection from infection with different bacterial species. The nose is usually exposed to infections with different pathogen germs, which may affect also the fibers of the trigeminal and olfactory nerves found in the nasal cavity ${ }^{14}$. The olfactory nerve may be a more susceptible route because primary olfactory neurons are in direct contact with the external environment. It was shown that olfactory ensheathing cells and Schwann cells are involved in responding to the bacterial invasion, human OECs being able to phagocyte E. coli $^{14}$. OECs are an essential component of the innate immune response against bacterial aggression of the central nervous system via olfactory nerve, being able to detect and respond to bacterial challenge via the synthesis of nitric oxide ${ }^{15}$. These findings may contribute to the understanding of the mechanisms of different infections, and also can help in evaluating the perspective of using olfactory cells for regenerative therapies after neural damage ${ }^{14}$.

\section{CHARACTERISTICS OF OLFACTORY ENSHEATING CELLS}

The glial cells of the olfactory bulb were first identified by the histologists Golgi and Blanes at the end of the nineteenth century ${ }^{16}$, being thought to be included in Schwann cells family, but further studies demonstrated that they are a distinct glial cell type ${ }^{16,}$ 17,18 . In the early studies another terminology was used, defining two different terms depending of the source of cell isolation: olfactory bulb ensheathing cells -cells isolated from olfactory bulbs and olfactory nerve ensheathing cells. This classification is no longer relevant, in present the proper term for these cells being olfactory ensheathing cells (olfactory ensheathing glia) ${ }^{18}$. Olfactory ensheathing glia shares some properties with Schwann cells and astrocytes ${ }^{6}$, although because of their different pattern of markers and properties are classified as a different type of glial cells. There are differences in the interactions between astrocytes and either Schwann cells or OECs observed in tissue culture models and reveal one aspect of the complex biology of creating regenerationpromoting environments by cell transplantation while transplanted OECs have favourable properties compared to transplanted Schwann cells ${ }^{19}$.

OECs present a large heterogeneity in culture with respect to their cellular morphology and expressed molecules. Studies made in vivo identified a series of markers expressed by OECs that are useful for their identification ${ }^{1}$. For research purpose, the cells are usually isolated from the olfactory bulb of rodents, but OECs have also been purified from dogs, pigs, primates and humans. A dissection is practiced to the olfactory bulb, separating the components and isolation 
of the nerve fiber layer. OECs from both peripheral and central olfactory tissue can be purified and cultured $^{18}$. A variety of purification methods have been implemented, including the sorting of cells based on their expression of different molecules, such as sulfatide $\mathrm{O} 4$, and not expressing others, like galactocerebroside $^{20}$, immunopurification techniques, selection of positive cells for different receptors, and methods based on differential adhesion of OECs. It was demonstrated that OECs are very heterogeneous regarding their morphology and markers expressed in culture, also after purification ${ }^{21,22,23}$. Feron et al. established a clinical therapeutic protocol: olfactory ensheathing cells are prelevated from nasal biopsies, cells were grown and purified in vitro and injected into the region of damaged spinal cord of selected patients ${ }^{24}$.

In order to use OECs for cell therapy, it is necessary to produce enough purified cells quickly prior to transplantation. This may take several weeks following cell purification. It is important to know that if the purification of OECs from the olfactory mucosa or bulb is not carried out, a large number of other cell types ("contaminating cell types") will be isolated and propagated in culture, including fibroblast-like cells, mesenchymal stem cells, connective cells, immune cells and pericytes ${ }^{25,26,27}$. The conditions of cell culture are very important in obtaining rapid and stable proliferation and also to maintain cellular morphological characteristics and antigenic properties ${ }^{18}$.

\section{THERAPEUTIC IMPLICATIONS OF OLFACTORY ENSHEATING CELLS}

\section{Central Nervous System Disorders}

Central nervous system-brain and spinal cord represent a unique structure, with limited capacity of repair after different injuries, with consequences in severe functional disability. Researchers are looking for "the ideal cell type" in order to transplant it in the nervous system and obtain neural recovery; transplantation of autologous cells represents an advantage, to avoid immunological aspects involved and also the risk of transmissible diseases ${ }^{28}$. Based on its capacity for regeneration, olfactory tissue represent a promising donor site of cells. Scientists are already using olfactory cells, in animal models or early clinical studies, for the treatment of central nervous system damage, from different injuries and neurodegenerative diseases ${ }^{27}$. The possibility of using OECs for neural repair after injuries demonstrates an important property of those cells: the ability to manifest a substantial plasticity ${ }^{29}$.

\section{Spinal Cord Injuries}

Different cell types have been used in attempting to treat spinal cord injuries, including Schwann cells,
OECs, neural stem/progenitor cells, fate-restricted neural and glial precursors and bone marrow stromal cells $^{30}$. Due to the ability of OECs to support continual outgrowth of olfactory receptor axons throughout life, their potential benefits in cell transplantation to repair the injured spinal cord have been the subject of a considerable amount of research over the past two decades. OECs can be harvested from the olfactory bulb or from olfactory mucosa. The donor site morbidity is higher for the olfactory bulb source (bulb source is frequently used in animal models worldwide) and less invasive for olfactory mucosa, this approach being better and recommendable as cell source for autologous transplantation ${ }^{31}$. Iwatsuki transplanted the whole olfactory mucosa containing olfactory epithelium, lamina propria, and OECs and showed improvement in functional recovery after spinal cord injury ${ }^{32}$.

There are several clinical trials that support the use of OECs based therapy for spinal cord lesions ${ }^{33}$. Usually, biopsies are taken from the olfactory mucosa, than olfactory ensheathing cells are cultured from these biopsies for 4-10 weeks. After appropriate testing, transplantation of the cells into the injured spinal cord is performed; the procedure requires microscopic control and using of a purposedesigned injector device ${ }^{24}$. A phase I/IIa trial transplantation from Australia, including a small number of patients, propose the use of autologous olfactory ensheathing cells transplanted into the injured spinal cord as a feasible and safe procedure at 3 years post-implantation, with promising functional recovery $^{34}$. Similar results were published by Tabakow et al., after a phase I-clinical trial including six patients with chronic thoracic paraplegia ${ }^{35}$. Zheng and Liu analyzed the nerve recovery after OECs transplantation for chronic spinal cord injury, using sympathetic skin response, a test that is a simple, safe, noninvasive electrophysiological detection method, and can objectively assess autonomic nerve functions. Their study demonstrated improvements in sympathetic skin responses, findings indicating that olfactory ensheathing cell transplantation restore motor, sensory and autonomic nerve functions in patients with chronic spinal cord injury ${ }^{36}$.

Transplantation of olfactory ensheathing cells into the injured spinal cord demonstrates neuroprotective effects and promotes functional recovery, OECs exerting modulator effects, decreasing inflammatory reaction, probably by earlier, higher and shorter duration of microglia/macrophage and astrocyte responses after injury ${ }^{37}$. As was shown, olfactory ensheathing cells transplantation is a novel therapeutic possibility for patients with injuries of the spinal cord, with a large number of scientific publications reporting promising results of this treatment option ${ }^{38}$. 


\section{Brain Injuries}

Olfactory ensheathing cells may have a potential therapeutic role in recovery after ischemic insults of the brain, as is seen in stroke, with the ability to protect the white matter from ischemic injury. In a rat model of ischemic stroke, OECs transplantation reduced the infarct volume, decreased mortality and improved neurological deficits, indicating that the remyelination and axonal regeneration in OEC transplanted rat were significant ${ }^{39}$. In animal model-stroke was shown that the olfactory cells transplanted intracerebral can determine functional recovery of neural tissue and also a reversibility of destructive process ${ }^{40,41}$. Several studies demonstrated that olfactory ensheathing cells are able of synthesize neurotrophic factors for the transplanted neural cells promoting their long-term survival. The results obtain in rodent model of cognitive dysfunction confirm the hypothesis that co-transplantation of olfactory ensheathing cells and neural progenitor cells may be a better approach for functional restoration ${ }^{42}$.

\section{Neurodegenerative Diseases}

Amyotrophic lateral sclerosis (ALS) is a degenerative disease determining the loss of motor neurons in the motor cortex, the brain stem, and the spinal cord. It represents a very severe condition, decreasing life-expectancy and affecting quality of life. A clinical study projected to analyze if transplantation of fetal olfactory ensheathing cells will influence the evolution of disease in patients with ALS, showed that transplantation of olfactory cells are capable to slow, based on clinical findings, the rate of progression within the first four months after transplantation ${ }^{43}$. The authors of another study found that seriate treatment involving multiple injection is safe and feasible, despite the fragility of spine in ALS with observation of functional improvement after treatment ${ }^{44,45}$.

There are different experimental models using OECs for Parkinson's disease. The co-transplatation of olfactory cells from the olfactory bulb and the fetal ventral mesencephalic cells in striatal region, in 6-hydroxydopamine(6-OHDA)-lesioned murine model of Parkinson's disease suggested good outcomes for functional restoration, enhancing the survival of the dopaminergic neurons and promoting striatal reinnervation ${ }^{46}$.

\section{Peripheral Nervous System Injuries}

Peripheral nerves injuries occur frequently after traumatic events, often affecting young people, with serious functional repercussion over the quality of life of the individual. Regeneration of the axons is better in the peripheral nervous system than in the central nervous system, but it is never complete and the searching for methods to improve peripheral nerve regeneration is an important research area. Different cell types, Schwann cells, OECs derived from the olfactory mucosa or olfactory bulb, stromal cells from bone marrow- or adipose tissue-derived cells, have been used in order to repair peripheral nerve injuries $^{42}$. In association with microsurgical repair of a rat sciatic nerve, the application of OECs at the time of nerve injury enhances regeneration and improves functional outcome. OECs have been used to promote peripheral nerve regeneration, due to their properties: ability to migrate and to penetrate glial scars, increasing axon regeneration, myelination, and nodal formation ${ }^{47}$.

After peripheral nerve injury, in cases of complete sections, axonal regrowth may be impaired by misrouting, aspect studied by Guerout and his team in experimental models of transection of facial and laryngeal nerve. Complete section of the recurrent laryngeal nerve determines abnormal regrowth of axons with clinical evidence being synkinesis of the vocal cords. In the model described by the authors, after section and anastomosis of the recurrent laryngeal nerve, it was showed that OECs transplantation efficiently increases axonal regrowth, EMG analyses proving richness and synchronization of muscular activities and histological findings, based on quantification of the number and characteristics of myelinated fibers after toluidine blue coloring ${ }^{2}$.

\section{POTENTIAL IMMUNOMODULATORY ROLE OF OECS}

The immune system has a crucial role in promoting wound healing. Immune cells are involved in innate immunity, inflammation being the fundamental regulators of tissue repair and wound healing, based on their secretion of growth factors and cytokines ${ }^{48}$.

Researchers try to understand better the wound healing process, with its mechanisms of regulation by different factors of the immune system in order to elucidate the pathogenesis of the wide spectrum of immune and inflammatory disorders ${ }^{48}$.

Recent research suggests that OECs have immunomodulatory properties, being able to reduce the intensity of neuroinflammation when they are transplanted into damaged areas of the central nervous system. The anti-inflammatory effects of the cultivated OECs may appear due to the presence, as soluble factor, of insulin-like growth factor 1 (IGF-1). Also, it seems that OECs, in their interactions with astrocytes, moderate the inflammatory activation of astrocytes, being able to overcome the harmful effects of the glial scar $^{49}$.

Multiple sclerosis and others demyelinating diseases affecting the central nervous system, are inten- 
sively studied, representing invalidating disorders affecting the patients as well as their families. Recently, investigators with experience in cell therapy recommended the olfactory ensheathing cells isolated from olfactory mucosa as a valuable option of treatment for these diseases, based on the potential immunomodulatory properties and improvement of myelination. Comparing with other cell types studied for the capacity to promote remyelination, it has been shown that olfactory precursor cells are the most efficient cells at recovery the demyelinated axons ${ }^{50}$. Regeneration of myelin sheath following OECs transplant is like sheath formation after combined transplantation of Schwann cells and astrocytes ${ }^{51,52}$. Following OECs transplant, a relatively thick myelin is formed, accompanied with a large amount of cytoplasm and nucleus surrounding the demyelinating axons, a result from a single OECs forming myelin sheath for a single neuraxon segment $t^{53}$. Further studies are needed in terms of understanding the immunoregulatory role for the inflammatory demyelinating disease.

\section{CONCLUSIONS}

Olfactory ensheathing cells are distinct glial cells that possess unique characteristics that make them an eligible source in developing therapeutic strategies for neural injuries in central nervous system, peripheral nervous system, neurodegenerative diseases such as Amyotrophic Lateral Sclerosis and Parkinson Disease, and also demyelinating disorders. An emerging field is represented by the implication of the OECs in modulation of the immune response. Thus, further studies are needed for a thorough and extensive understanding of the biological and molecular properties of OECs, interactions with other cell populations and their mechanism of action in different pathologies, in order to ensure the clinical efficacy after cell transplantation.

Acknowledgement: This paper is supported by the Sectoral Operational Programme Human Resources Development (SOP HRD,) financed from the European Social Fund and by the Romanian Government under the contract number POSDRU/159/1.5/S/137390

\section{REFERENCES}

1. Ramón-Cueto A., Avila J. - Olfactory ensheathing glia: properties and function. Brain Res Bull., 1998;46(3):175-87.

2. Guerout N., Paviot, A., Bon-Mardion N., Honoré A., OBongo R., Duclos C. - Transplantation of Olfactory Ensheathing Cells to Evaluate Functional Recovery after Peripheral Nerve Injury. J. Vis. Exp., 2014;23(84):e50590. doi: 10.3791/50590.

3. Su Z, He C. - Olfactory ensheathing cells: biology in neural development and regeneration. Prog Neurobiol., 2010;92(4):517-32.

4. Roet K.C., Verhaagen J. - Understanding the neural repair-promoting properties of olfactory ensheathing cells. Exp. Neurol., 2014;261:594609.

5. Ekberg J.A., St. John J.A. - Crucial roles for olfactory ensheathing cells and olfactory mucosal cells in the repair of damaged neural tracts. Anat. Rec., 2014;297(1):121-128.

6. Doucette R. - Glial influences on axonal growth in the primary olfactory system. Glia, 1990;3(6):433-449.

7. Mackay-Sim A., Kittel P. - Cell dynamics in the adult mouse olfactory epithelium: A quantitative autoradiographic study. J. Neurosci., 1991;11(4):979-984.

8. De Carlos J.A., Lopez-Mascaraque L., Valverde F. - Early olfactory fiber projections and cell migration into the rat telencephalon. Int J Dev Neurosci., 1996;14:853-866

9. Windus L.C., Chehrehasa. F., Lineburg K.E, Claxton C., Mackay-Sim A., Key B., St John J.A. - Stimulation of olfactory ensheathing cell motility enhances olfactory axon growth. Cell. Mol. Life. Sci., 2011;68(19):32333247.

10. Chehrehasa F., Windus L.C., Ekberg J.A., Scott S.E., Amaya D., MackaySim A., St John J.A. - Olfactory glia enhance neonatal axon regeneration. Mol. Cell. Neurosci., 2010;45(3):277-288.

11. Au W.W., Treloar H.B., Greer C.A. - Sublaminar organisation of the mouse olfactory bulb nerve layer. J. Comp. Neurol., 2002;46:68-80.

12. Ekberg J.A., Amaya D., Mackay-Sim A., St John J. A. - The migration of olfactory ensheathing cells during development and regeneration. Neurosignals, 2012;20(3):147-158.

13. Zhu Y., Cao L., Su Z., Mu L., Yuan Y., Gao L., Qiu Y., He C. - Olfactory ensheathing cells: Attractant of neural progenitor migration to olfactory bulb. Glia, 2010;58(6):716-729.

14. Panni P., Ferguson I.A., Beacham I., Mackay-Sim A., Ekberg J.A., St John J.A. - Phagocytosis of bacteria by olfactory ensheathing cells and Schwann cells. Neurosci Lett., 2013;28(539):65-70.

15. Harris J.A., West A.K., Chuah M.I. - Olfactory ensheathing cells: nitric oxide production and innate immunity. Glia, 2009;57(16):1848-1857.

16. Golgi C. - Sulla fina anatomia del bulbi olfattori. Ti Rivista Sperimentale di Freniatria., 1875;1:403-425.

17. Blanes T. - Sobre algunos puntos dudosos de la estructura del bulbo olfatorio. Rev Trim Micrograf., 1898;3:99-127.

18. Higginson J.R., Barnett S.C. - The culture of olfactory ensheathing cells (OECs)-a distinct glial cell type. Exp Neurol., 2011;229(1):2-9.

19. Lakatos A., Barnett S.C., Franklin R.J. - Olfactory ensheathing cells induce less host astrocyte response and chondroitin sulphate proteoglycan expression than Schwann cells following transplantation into adult CNS white matter. Exp Neurol., 2003;184(1):237-246.

20. Barnett S.C., Hutchins A.M., Noble M. - Purification of olfactory nerve ensheathing cells from the olfactory bulb. Dev Biol., 1993;155:337-350.

21. Moreno-Flores M.T., Díaz-Nido J., Wandosell F., Avila J. - Olfactory Ensheathing Glia: Drivers of Axonal Regeneration in the Central Nervous System? J Biomed Biotechnol., 2002;2(1):37-43.

22. Ramon-Cueto A., Nieto-Sampedro M. - Glial cells from adult rat olfactory bulb: immunocytochemical properties of pure cultures of ensheathing cells. Neuroscience, 1992;47:213-220.

23. Nash H.H., Borke R.C., Anders J.J. - New method of purification for establishing primary cultures of ensheathing cells from the adult olfactory bulb.Glia, 2001;34:81-88.

24. Feron F., Perry C., Cochrane J., Licina P., Nowitzke A., Urquhart S. Autologous olfactory ensheathing cell transplantation in human spinal cord injury. Brain, 2005;128:2951-2960. 
25. Barnett S.C., Chang L. - Olfactory ensheathing cells and CNS repair: going solo or in need of a friend? Trends Neurosci., 2004;27:54-60.

26. Tomé M., Lindsay S.L., Riddell J.S., Barnett S.C. - Identification of nonepithelial multipotent cells in the embryonic olfactory mucosa. Stem Cells, 2009;27:2196-2208.

27. Lindsay S.L., Riddell J.S., Barnett S.C. - Olfactory mucosa for transplantmediated repair: a complex tissue for a complex injury? Glia, 2010;58:125-134.

28. Viktorov I.V., Savchenko E.A., Ukhova O.V., Alekseyeva N.Y., Chekhonin V.P. - Multipotent stem and progenitor cells of the olfactory epithelium. Bull Exp Biol Med., 2006;142(4):495-502.

29. Vincent A.J., West A.K., Chuah M.I. - Morphological and functional plasticity of olfactory ensheathing cells. J Neurocytol., 2005;34(1-2):65-80.

30. Chou R.H., Lu C.Y., Wei-Lee Fan J.R., Yu Y.L., Shyu W.C. - The potential therapeutic applications of olfactory ensheathing cells in regenerative medicine. Cell Transplant., 2014;23(4-5):567-571.

31. Mayeur A., Duclos C., Honoré A., Gauberti M., Drouot L., do Rego J.C., Bon-Mardion N., Jean L., Vérin E., Emery E., Lemarchant S., Vivien D., Boyer O., Marie J.P., Guérout N. - Potential of olfactory ensheathing cells from different sources for spinal cord repair. PLoS One., 2013 Apr 24;8(4):e62860. doi: 10.1371/journal.pone.0062860.

32. Iwatsuki K., Yoshimine T., Kishima H., Aoki M., Yoshimura K., Ishihara M., Ohnishi Y., Lima C. - Transplantation of olfactory mucosa following spinal cord injury promotes recovery in rats. Neuroreport., 2008;19(13):1249-1252.

33. Mackay-Sim A., St John J.A. - Olfactory ensheathing cells from the nose: clinical application in human spinal cord injuries. Exp Neurol., 2011;229(1):174-180.

34. Mackay-Sim A., Féron F., Cochrane J., Bassingthwaighte L., Bayliss C., Davies W., Fronek P., Gray C., Kerr G., Licina P., Nowitzke A., Perry C., Silburn P.A., Urquhart S., Geraghty T. - Autologous olfactory ensheathing cell transplantation in human paraplegia: a 3-year clinical trial. Brain, 2008;131 (Pt 9):2376-2386.

35. Tabakow P., Jarmundowicz W., Czapiga B., Fortuna W., Miedzybrodzki R., Czyz M., Huber J., Szarek D., Okurowski S., Szewczyk P., Gorski A., Raisman G. - Transplantation of autologous olfactory ensheathing cells in complete human spinal cord injury. Cell Transplant., 2013;22(9):15911612.

36. Zheng Z., Liu G., Chen Y., Wei S. - Olfactory ensheathing cell transplantation improves sympathetic skin responses in chronic spinal cord injury. Neural Regeneration Research., 2013;8(30):2849-2855.

37. López-Vales R., García-Alías G., Forés J., Vela J.M., Navarro X., Verdú E. - Transplanted olfactory ensheathing cells modulate the inflammatory response in the injured spinal cord. Neuron Glia Biol., 2004;1(3):201209.

38. Leng Z., He X., Li H., Wang D., Cao K. - Olfactory ensheathing cell transplantation for spinal cord injury: An 18-year bibliometric analysis based on the Web of Science. Neural Regeneration Research., 2013;8(14):12861296

39. Shi X., Kang Y., Hu Q., Chen C.,Yang L., Wang K.,Chen L., Huang H., Zhou C. - A long-term observation of olfactory ensheathing cells trans- plantation to repair white matter and functional recovery in a focal ischemia model in rat. Brain Res., 2010;1317:257-267.

40. Shyu W.C., Liu D.D., Lin S.Z., Li W.W., Su C.Y., Chang Y.C., Wang H.J., Wang H.W., Tsai C.H., Li H. - Implantation of olfactory ensheathing cells promotes neuroplasticity in murine models of stroke. J. Clin. Invest., 2008;118(7):2482-2495.

41. Chou R.H., Lu C.Y., Wei-Lee Fan J.R., Yu Y.L., Shyu W.C. - The potential therapeutic applications of olfactory ensheathing cells in regenerative medicine. Cell Transplant., 2014;23(4-5):567-571.

42. Srivastava N., Seth K., Khanna V.K., Ansari R.W., Agrawal A.K. - Longterm functional restoration by neural progenitor cell transplantation in rat model of cognitive dysfunction: co-transplantation with olfactory ensheathing cells for neurotrophic factor support. Int J Dev Neurosci., 2009;27(1):103-110.

43. Huang H., Chen L., Xi H., Wang H., Zhang J., Zhang F., Liu Y. - Fetal olfactory ensheathing cells transplantation in amyotrophic lateral sclerosis patients: A controlled pilot study. Clin. Transplant., 2008;118(7):24822495.

44. Chen L., Chen D., Xi H., Wang Q., Liu Y., Zhang F., Wang H., Ren Y., Xiao J., Wang Y., Huang H. - Olfactory ensheathing cell neurorestorotherapy for amyotrophic lateral sclerosis patients: benefits from multiple transplantations. Cell Transplant., 2012;21 Suppl 1:S65-77.

45. Kim C., Lee H.C., Sung J.J. - Amyotrophic lateral sclerosis - cell based therapy and novel therapeutic development. Exp Neurobiol., 2014;23(3):207-214.

46. Agrawal A.K., Shukla S., Chaturvedi R. K., Seth K., Srivastava N., Ahmad A., Seth P.K. - Olfactory ensheathing cell transplantation restores functional deficits in rat model of Parkinson's disease: A cotransplantation approach with fetal ventral mesencephalic cells. Neurobiol. Dis., 2004;16(3):516-526.

47. Radtke C., Aizer A.A., Agulian S.K., Lankford K.L., Vogt P.M., Kocsis J.D. - Transplantation of olfactory ensheathing cells enhances peripheral nerve regeneration after microsurgical nerve repair. Brain Res., 2009;1254:10-17.

48. Harty M., Neff A.W., King M.W., Mescher A.L. - Regeneration or scarring: an immunologic perspective. Dev Dyn., 2003;226:268-279.

49. Chuah M.I., Hale D.M., West A.K. - Interaction of olfactory ensheathing cells with other cell types in vitro and after transplantation: glial scars and inflammation. Exp Neurol., 2011;229(1):46-53.

50. Franklin R.J., French-Constant C. - Remyelination in the CNS: from biology to therapy. Nat Rev Neurosci., 2008;9:839-855.

51. Shields S.A., Blakemore W.F., Franklin R.J. - Schwann cell remyelination is restricted to astrocyte-deficient areas after transplantation into demyelinated adult rat brain. J. Neurosci. Res., 2000;60(5):571-578.

52. Oudega M., Xu X.M. - Schwann cell transplantation for repair of the adult spinal cord. J. Neurotrauma, 2006;23(3-4):453-467.

53. Wu W., Lan Q., Xu J., Zhu A., Fang W., Jiang Y, Hui G. - Transplantation of olfactory ensheathing cells for treatment of inflammatory demyelinating diseases in the central nervous system, Scientific Research and Essays, 2010;5(23):3676-3681. 